\title{
Determinación y análisis de la tenacidad a la fractura en materiales compuestos laminados fibra-metal
}

\section{Determination and analysis of fracture toughness on fiber-metal laminates composite materials}

Eduardo Asta ${ }^{1}$, Francisco Cambiasso ${ }^{1}$, Juan Balderrama ${ }^{1}$, Juan Ríos ${ }^{1}$

\author{
${ }^{1}$ Laboratorio de Ensayo de Materiales, Facultad Regional Haedo, Universidad Tecnológica Nacional, GIMF/FRH/UTN, \\ Calle París, n. 532, Haedo, Buenos Aires, Argentina. \\ e-mail: asta.eduardo@gmail.com,fcambiasso@frh.utn.edu.ar, rios@cnea.gov.ar
}

\section{RESUMEN}

La determinación de la tenacidad a la fractura en materiales compuestos híbridos, laminados fibra-metal, FML (Fiber Metal Laminates) resulta muy importante para poder realizar un análisis de integridad estructural que permita estimar la tolerancia al daño y la resistencia remanente del material frente a la propagación de una fisura. Dicha evaluación debe realizarse tanto en estructuras aeronáuticas como navales que utilizan compuestos FML del tipo CARALL (CArbon Reinforced Aluminum Laminates).

En este trabajo se presenta la determinación experimental de la tenacidad a la fractura en términos de los parámetros $J$ y CTOD utilizando probetas compactas $\mathrm{C}(\mathrm{T})$ en dos tipos de material compuesto laminado con láminas de aluminio 6061T0 en un caso y láminas de aluminio 1050 en otro y fibras de carbono NCT-301, embebidas en resina epoxi, para ambos materiales. Los resultados indican que la adaptación de las técnicas de ensayo de tenacidad a la fractura elasto-plástica, aplicando la norma ASTM E1820, son aceptables para la determinación de la tenacidad a la fractura J en compuestos del tipo CARALL. Dicha caracterización es necesaria para el desarrollo de un programa de evaluación de daño tolerable tanto a nivel de diseño como para un análisis de significación de defectos a partir de resultados de ensayos no destructivos sobre este tipo de materiales compuestos.

Palabras clave: compuestos, tenacidad, fractura, fisura, delaminación.

\begin{abstract}
The determination of fracture toughness in hybrid composite materials, fiber-metal laminates(FML) is very important in order to make a structural integrity analysis that allows estimating damage tolerance and the remaining strength of the material against crack propagation. This evaluation should be carried out in both aeronautical and naval structures that use FML composites, CARALL type (CArbon Reinforced Aluminum Laminates).

This paper presents the experimental determination of fracture toughness in terms of parameters $J$ and $C T O D$ using compact $\mathrm{C}(\mathrm{T})$ specimens in two types of composite material laminated with 6061-T0 aluminum sheets in one case and sheets 1050 aluminum in another and carbon fibers NCT-301, embedded in epoxy resin, for both materials. The results indicate that the use of the elasto-plastic fracture toughness test techniques, applying ASTM E1820, are acceptable for the determination of the fracture toughness J in CARALL type materials. Such characterization is necessary for the development of a tolerable damage assessment program both at the design level and for a defect significance analysis based on the results of non-destructive tests on this type of composite materials.
\end{abstract}

Keywords: composites, toughness, fracture, cracks, delamination. 


\section{INTRODUCCIÓN}

Los materiales compuestos híbridos, laminados fibra-metal, (Fiber Metal Laminates FML), consisten en láminas delgadas de aluminio alternadas con capas de fibras de carbono unidireccionales embebidas en una matriz de resina epoxi [1]. Los compuestos FML son materiales livianos, con elevada resistencia a la propagación de fisuras, resistencia al daño acumulado y baja absorción de humedad. Tienen aplicación tanto en la industria aeroespacial como naval y la posibilidad de aplicación en diferentes estructuras vehiculares [2]. En estos materiales la propagación de fisuras tiene lugar mediante el mecanismo denominado "puenteo de fibra" (fiber-bridging) [3].

Cuando el extremo de una fisura se propaga en forma perpendicular a la fibra tal propagación se realiza preferentemente a través de las láminas de aluminio, acompañada por una delaminación controlada, entre las láminas metálicas y la matriz polimérica. Esto causa poco daño en las fibras que permanecen mayoritariamente intactas detrás del extremo de la fisura. Este mecanismo es clave para reducir el factor de intensidad de tensiones en el extremo de la fisura, disminuyendo su velocidad de propagación.

El diseño de este tipo de estructuras incorpora el concepto de daño tolerado, que requiere el conocimiento de las características de propagación por fatiga de los componentes o partes estructurales y además se necesita conocer el comportamiento de tenacidad a la fractura para establecer las condiciones de tamaño crítico de fisuras bajo diferentes condiciones de carga y resistencia residual. Sin embargo, la resistencia residual, para la condición de falla, es un evento complejo que involucra múltiples mecanismos de falla, tales como deformación plástica, delaminación estática, crecimiento de fisura en la lámina de metal y rotura de fibra $[4,5]$.

En modelos adoptados por varios autores la tenacidad a la fractura en compuestos FML ha sido determinada utilizando la mecánica de fractura lineal-elástica a partir de las normas generales de ensayo, por ejemplo, la norma ASTM E-561 $[6]$.

Cuando se obtiene la respuesta del material en un registro carga-desplazamiento, con diferentes tipos de probetas normalizadas, aparece un comportamiento que se aparta de la linealidad con indicios de cierto crecimiento estable de fisura e inestabilidades del tipo "pop-in" o inmediatamente seguido de la condición de fractura.

Esto conduce, por analogía con los materiales metálicos, a la propuesta de utilización de las técnicas de ensayo de la mecánica de fractura elasto-plástica (Integral J y CTOD) en compuestos FML, aplicando la metodología de la norma ASTM E-1820 para poder determinar la tenacidad a la fractura crítica [7,8].

Estos criterios de tenacidad a la fractura con sus técnicas de ensayo presentan limitaciones respecto de la representatividad para determinar la resistencia residual en materiales compuestos FML que presentan anisotropía mecánica, espesores delgados, dependencia de la geometría y un mecanismo de fractura que asocia la propagación metálica y delaminación entre la lámina de metal y las capas de resina reforzada con fibras no metálicas.

En este trabajo se presenta una determinación experimental de la tenacidad a la fractura en términos del parámetro J utilizando probetas $\mathrm{C}(\mathrm{T})$ de acuerdo con ASTM en material FML con láminas metálicas de aluminio y fibras de carbono embebidas en resina epoxi.

\section{MATERIALES Y MÉTODOS}

Se han ensayado con la metodología de la norma ASTM E-1820 (8) dos tipos de compuestos FML fabricados con láminas metálicas de aluminio 6061-O y 1050, en ambos casos utilizando fibra de carbono NCT-301 unidireccional, impregnada en resina epoxi. Previamente se han determinado las propiedades mecánicas por medio de ensayos de tracción ambos compuestos FML.

\subsection{Materiales}

En las Tablas 1 y 2 se presentan respectivamente las propiedades mecánicas de los aluminios y de la fibra de carbono utilizados en la fabricación del compuesto FML (9).

El laminado para uso experimental ha sido elaborado en placas cuadradas de $120 \mathrm{~mm}$ de lado según las secuencias de apilamiento o arreglo representada por medio de la indicación $m / n(m=n+1)$, siendo $m$ la cantidad de capas de aluminio y $\mathrm{n}$ la cantidad de capas de fibras impregnadas en resina. En este trabajo se ha utilizado un arreglo 7/6 (Al-90-Al-0-Al-90Al-90-Al-0-Al-90-Al) con Al 6061-O, otro 5/4 también con Al 6061-O, siendo $0^{\circ}$ y 90 la orientación de la fibra de carbono respecto a la dirección de laminación de la aleación de aluminio, y un arreglo similar 4/3 elaborado con Al 1050. El conformado del compuesto se realizó con saco de vacío y el curado en autoclave a una presión de 6 bar y a una temperatura de $136{ }^{\circ} \mathrm{C}$, tal como se puede observar en el registro de la Figura 1. 
Tabla 1: Propiedades mecánicas de los aluminios utilizados.

\begin{tabular}{c|c|c|c|c|c}
\hline Material & $\begin{array}{c}\text { Módulo de } \\
\text { elasticidad } E \\
(\mathbf{G P a})\end{array}$ & $\begin{array}{c}\text { Coeficiente } \\
\text { de Poisson } \\
\mathbf{v}_{\mathbf{x y}}\end{array}$ & $\begin{array}{c}\text { Tensión de } \\
\text { fluencia } \sigma_{\boldsymbol{f}} \\
\mathbf{( M P a )}\end{array}$ & $\begin{array}{c}\text { Resistencia a } \\
\text { la tracción } \\
\sigma \boldsymbol{r}(\mathbf{M P a})\end{array}$ & $\begin{array}{c}\text { Elongación } \\
\mathbf{e}(\%)\end{array}$ \\
\hline 1050 & 69 & 0,33 & 20 & 65 & 20 \\
\hline $6061-\mathrm{O}$ & 69 & 0,33 & 85 & 150 & 20 \\
\hline
\end{tabular}

Tabla 2: Propiedades mecánicas de la lámina de fibra de carbono con resina epoxi.

\begin{tabular}{|c|c|c|c|c|c|}
\hline \multirow{2}{*}{ Material } & \multicolumn{2}{|c|}{ Módulo de elasticidad, $E$} & \multirow{2}{*}{$\begin{array}{l}\text { Módulo de Corte, } \\
\qquad G_{12} \\
(\mathrm{GPa})\end{array}$} & \multicolumn{2}{|c|}{$\begin{array}{c}\text { Carga primera falla de lámina, } \\
\qquad P P F^{\star}\end{array}$} \\
\hline & $\begin{array}{l}E_{1}\left(0^{\circ}\right) \\
(\mathrm{GPa})\end{array}$ & $\begin{array}{c}E_{2}\left(90^{\circ}\right) \\
(\mathrm{GPa})\end{array}$ & & $\begin{array}{l}F P F_{1} \\
(\mathrm{kN})\end{array}$ & $\begin{array}{l}F P F_{2} \\
(k N)\end{array}$ \\
\hline NCT-301 & 129,4 & 8,0 & 4,28 & 18,6 & 1,25 \\
\hline
\end{tabular}

*FPF: first ply failure

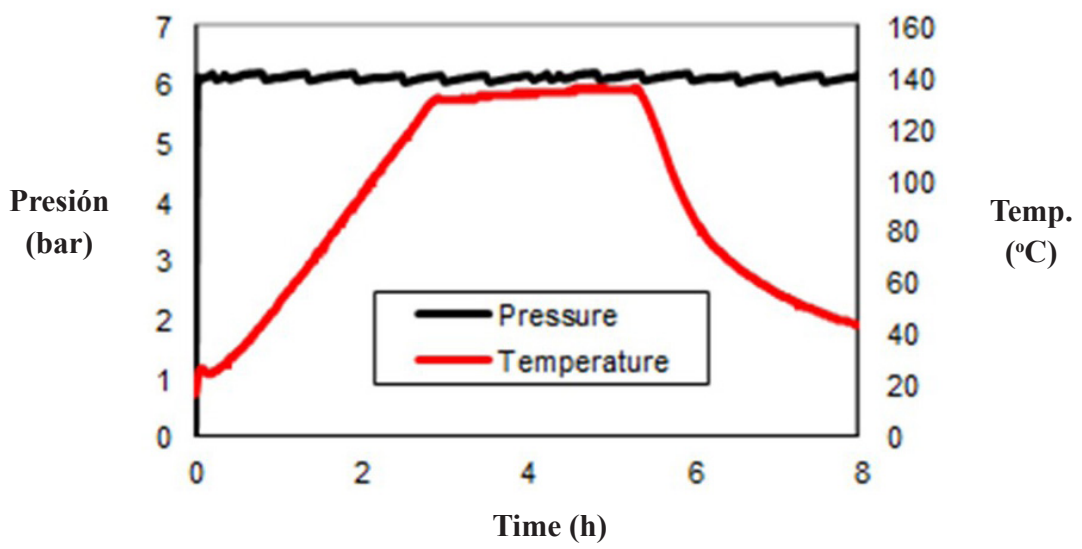

Figura 1: Ciclo de curado en autoclave, rampas de presión y temperatura.

\subsection{Obtención del parámetro integral $J$}

Para los ensayos de tracción han sido utilizadas probetas de sección rectangular constante y con láminas de aluminio aplicadas sobre los extremos con resina epoxi, con la finalidad de neutralizar los efectos de la fuerza de las mordazas del equipo de ensayo, tal como puede observarse en la Figura 2.

Se ha aplicado la metodología de ensayo elasto-plástico para la determinación de la tenacidad a la fractura en términos de la integral $J$, de acuerdo con los lineamientos de la norma ASTM 1820 [8].

Las probetas compactas $\mathrm{C}(\mathrm{T})[8,9]$, utilizadas para el ensayo de $J$, han sido fabricadas mediante mecanizado por control numérico y de acuerdo con lo especificado por la norma ASTM, tal como es indicado en la Figura 3.

A efectos de lograr una concentración de tensiones similar a la correspondiente a una pre-fisura por fatiga, tal como es requerido por la norma ASTM para la determinación de $J$ en materiales metálicos, la entalla de la probeta ha sido terminada con un radio de $0,15 \mathrm{~mm}$ en su extremo, tal como es mostrado en la Figura 4. 

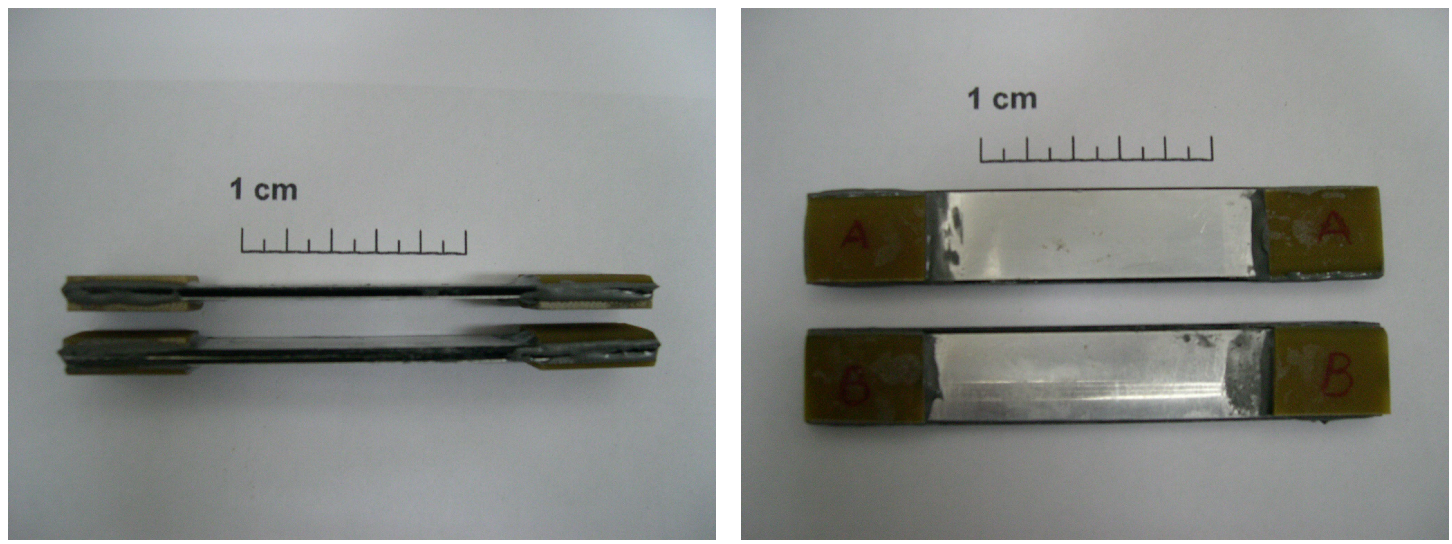

Figura 2: Probetas de tracción con apliques en los extremos.

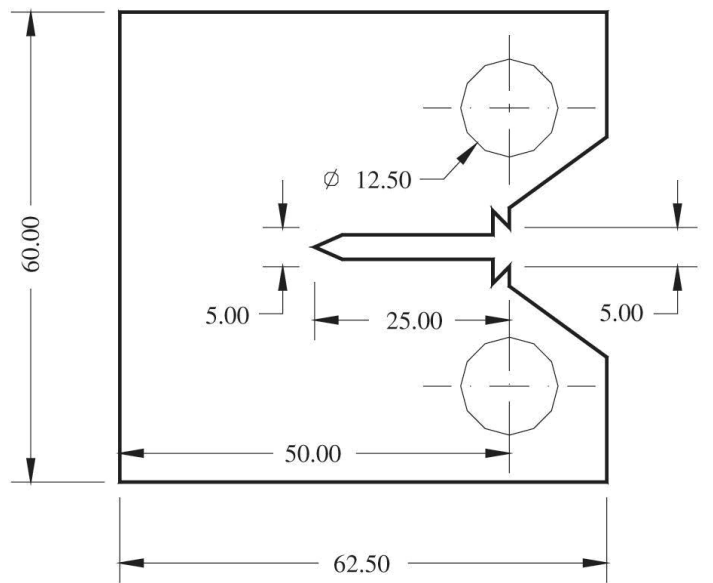

Figura 3: Probeta tipo $C(T) ; W=50 \mathrm{~mm}, \mathrm{a} 0=25 \mathrm{~mm}$



Figura 4: Modelo adoptado de punta de entalla de radio $0,15 \mathrm{~mm}$

Para la realización de los ensayos de tenacidad a la fractura se ha utilizado una máquina de ensayos servo-hidráulica donde la medición del desplazamiento en la línea de aplicación de la carga se ha efectuado por medio de un sensor de posición del cabezal. La integral $J$ se ha calculado de acuerdo con ASTM (8) como la suma de las componentes elástica y plástica de acuerdo con la ecuación (1):

$$
\mathrm{J}=\mathrm{J}_{\mathrm{el}}+\mathrm{J}_{\mathrm{pl}}
$$


La componente elástica, $J_{e l}$, es calculada con la ecuación (2), considerando condiciones de tensión plana con $a=a_{0}$ :

$$
J_{e l}=\frac{K^{2}}{E}
$$

El valor de $E$ en la ecuación (2) es el módulo elástico de un material ortótropo en la dirección de interés. En este caso, donde la entalla se encuentra ubicada sobre el plano principal, la solución se reduce a el caso isotrópico [7]. En consecuencia, el valor de $K$ ha sido calculado utilizando la resolución establecida en la norma ASTM [8]. La componente plástica de $J\left(J_{p l}\right)$ ha sido calculada de acuerdo con ASTM siguiendo la ecuación (3):

$$
\begin{gathered}
J_{p l}=\eta \frac{A_{p l}}{b_{0} B} \\
\eta=2+0,522 b_{0} / W
\end{gathered}
$$

Donde: $b_{0}=W$ - $a_{0}$ (ver Figura 3), $A_{p l}=$ área bajo la curva del registro carga $(P)$ - desplazamiento de la línea de aplicación de la carga $(d)$, en el punto final de inestabilidad de la carga o al primer "pop-in".

Los anexos de la norma ASTM E-1820 pueden ser utilizados para describir los detalles de la disposición experimental, la fabricación de probetas compactas $\mathrm{C}(\mathrm{T})$ y los desarrollos matemáticos utilizados a lo largo de este trabajo.

\section{RESULTADOS}

La Tabla 3 muestra la tensión de fluencia obtenida los compuestos CARALL en los ensayos de tracción.

La Tabla 4 resume los valores de tenacidad a la fractura obtenidos. Dado que en los análisis de daño tolerado es aplicada una metodología basada en el factor de intensificación de tensiones $K$, la Tabla 4 también contiene el valor de $K$ denominado crítico $(K)$. Dicho parámetro es obtenido a partir de $J_{c}$ y utilizando a ecuación (2) para el cálculo del valor de $K_{c}$.

Tabla 3: Tensión de fluencia obtenida en los ensayos de tracción.

\begin{tabular}{c|c}
\hline Material & $\boldsymbol{s}_{f}(\mathbf{M P a})$ \\
\hline CARALL (7/6) 6061 & 376 \\
\hline CARALL (4/3) 1050 & 347 \\
\hline
\end{tabular}

Tabla 4: Valores de tenacidad a la fractura críticos.

\begin{tabular}{l|c|c|c|c}
\hline Material & Probeta & $\begin{array}{c}\text { Espesor } \\
(\mathrm{mm})\end{array}$ & $\begin{array}{c}J_{c} \\
\left(\mathrm{~kJ} / \mathrm{m}^{2}\right)\end{array}$ & $\begin{array}{c}K_{c} \\
\left.(\mathrm{MPa} \mathrm{m})^{1 / 2}\right)\end{array}$ \\
\hline CARALL (7/6) 6061 & $\mathrm{C}(\mathrm{T})$ & 4,86 & 295 & 146 \\
\hline CARALL (5/4) 6061 & $\mathrm{C}(\mathrm{T})$ & 3,60 & 117 & 98 \\
\hline CARALL (4/3) 1050 & $\mathrm{C}(\mathrm{T})$ & 2,62 & 171 & 111 \\
\hline
\end{tabular}




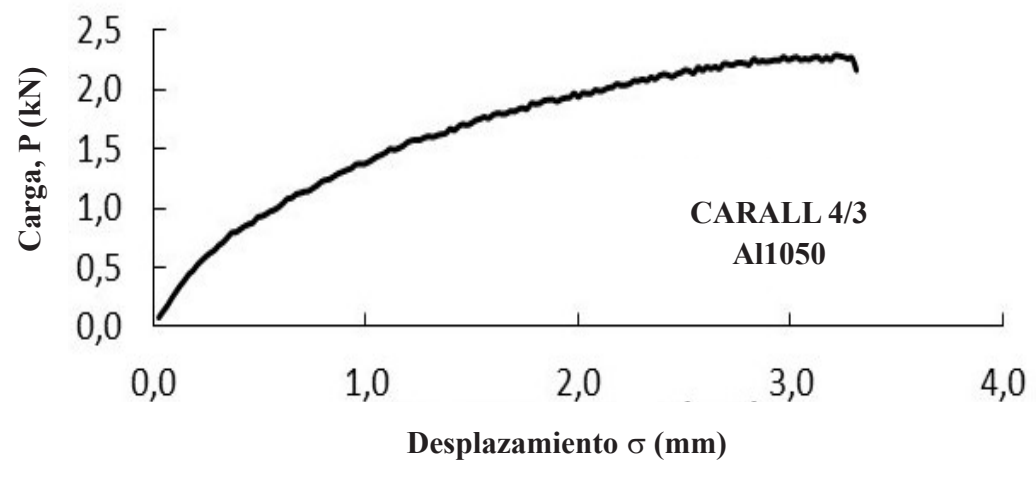

Figura 6: Registro de ensayo J, CARALL 4/3 Al 1050, espesor B = 2,62 mm.

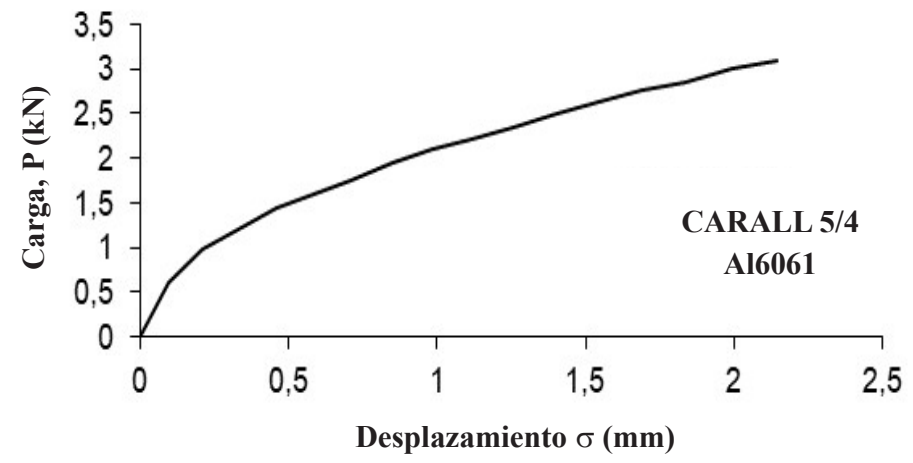

Figure 7: Registro de ensayo J, CARALL 5/4 Al 6061, espesor B = 3,60 mm.

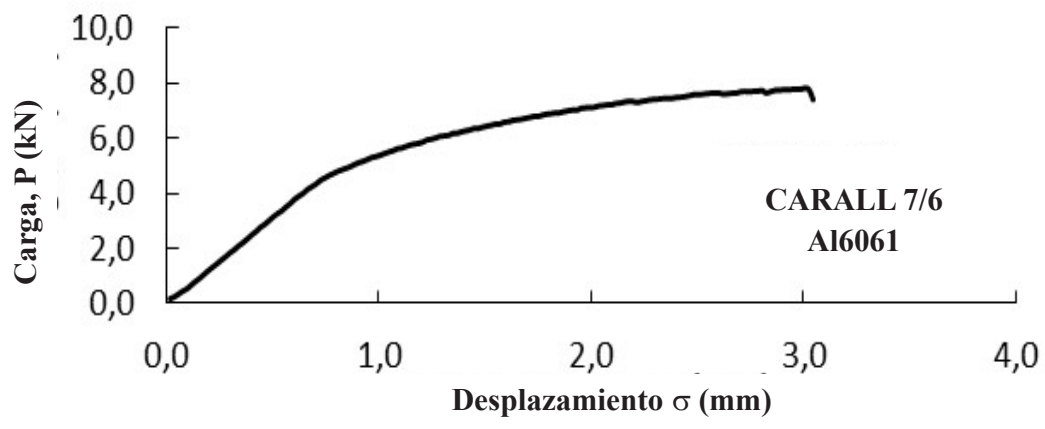

Figure 8: Registro de ensayo J, CARALL 7/6 Al 6061, espesor B =4,86 mm.

\section{DISCUSIÓN}

El comportamiento que se ha observado en los ensayos de fractura $J$, para ambos compuestos CARALL, muestra un aumento monótono de la carga hasta alcanzar el valor de carga máxima, seguido por la inestabilidad de fractura completa de ambos materiales compuestos, caracterizada por una caída brusca de carga sin recuperación, tal como es observado en los registros $P-d$ de las Figuras 6,7 y 8 respectivamente.

Antes de alcanzar el valor crítico de carga o la inestabilidad, se ha observado, a través del seguimiento óptico, un efecto de redondeo de la punta de entalla o muesca debido al proceso de deformación plástica en las láminas metálicas. No se ha observado una extensión o crecimiento estable de la fisura previa a la inestabilidad.

Teniendo en cuenta el comportamiento verificado del material a la fractura, con ausencia de pequeñas inestabilida- 
des del tipo "pop-in", se justifica la determinación aplicando el parámetro de tenacidad crítica a la fractura o inestabilidad crítica, de acuerdo con la norma ASTM [8]. El parámetro $J$ es calculado en el punto final de inestabilidad de las curvas $P$ - $d$ (Figuras 6, 7 y 8), utilizando las ecuaciones de $J$ para el método básico de cálculo, dicho punto es denominado $J_{Q c}$, un valor de $J$ crítico provisional calculado de acuerdo con la ecuación (1).

Los resultados provisionales de $J_{Q c}$ de los compuestos CARALL con Al 1050 y 6061 fueron sometidos a un análisis de validación, de acuerdo con lo requerido por la norma ASTM [8], con el fin de calificar los valores de $J_{Q c}$ como una medida de tenacidad a la fractura de inestabilidad crítica $\left(J_{c}\right)$, sin un significativo crecimiento estable de fisura, independiente de las dimensiones en plano $(W)$ pero que igualmente sería dependiente del espesor, aunque se cumplieran las condiciones de validación, debido al estado plano de tensión que se presenta en las probetas utilizadas. Los valores de $J_{O c}$ obtenidos para los materiales compuestos ensayados, bajo la metodología elasto- plástica, no han cumplido con las condiciones de calificación de acuerdo con ASTM E 1820 [8] para ser considerados como $J_{c}$, razón por la cual deberían permanecer como $J_{Q c}$. De esta manera, los valores de $J_{c}$ obtenidos (Tabla 4), junto con sus conversiones a valores de $K_{c}$ no pueden ser considerados insensibles a las dimensiones en el plano de la muestra, así como del espesor. En el análisis de validación es necesario tener en cuenta el hecho que el método de tenacidad a la fractura utilizado en este trabajo es, esencialmente, para materiales metálicos y que la propuesta de dicha metodología de ensayo para los compuestos FML está basada en la evidencia de un comportamiento a la fractura elasto-plástico de los mismos, considerando las limitaciones del estándar aplicado para tal fin.

Finalmente se han contrastado los resultados de tenacidad a la fractura para los compuestos CARALL evaluados con referencia a los otros compuestos FML del mismo tipo, informados en la literatura [7, 9, 10], habiéndose observado la consistencia con los mismos. El comportamiento no lineal de los materiales compuestos del tipo CARALL, mostrado experimentalmente en los ensayos de tenacidad a la fractura con probetas de ensayo ASTM C(T), ha permitido inferir la viabilidad de usar la metodología de tenacidad de fractura elasto-plástica. Sin embargo, un trabajo de validación exhaustivo y sistemático es necesario para predecir la resistencia remanente en elemento estructurales reales, a partir de los resultados experimentales con probetas de pequeño tamaño, así como para establecer un método estándar específico para la caracterización de la tenacidad a la fractura en compuestos FML.

Por otra parte, los valores de ensayo de tenacidad a la fractura informados en la Tabla 4 permiten cuantificar la influencia sobre las propiedades tanto del número de capas del apilamiento como del tipo de aluminio utilizado en el compuesto CARALL. En el compuesto con mejores propiedades mecánicas, en función de la aleación de aluminio utilizada para las capas metálicas del laminado, se verifica un incremento de la resistencia a la fractura, mientras que el mayor número de capas direcciona con idéntico resultado dicho comportamiento.

Para insertar dos ecuaciones seguidas se puede copiar la línea anterior y pegar en la próxima línea, el estilo se aplica automáticamente. Todas las ecuaciones deben ser numeradas secuencialmente.

\section{CONCLUSIONES}

Se ha determinado la tenacidad a la fractura crítica en compuestos FML del tipo CARALL fabricados con láminas de distintos aluminios y distintos arreglos con fibra de carbono de diversos espesores aplicando el método de ensayo de la integral $J$. Los valores obtenidos de $J_{c}$ son consistentes con los antecedentes de la bibliografía y se puede inferir la aplicabilidad de la metodología elasto-plástica para la caracterización a la fractura de compuestos FML, considerando las limitaciones analizadas.

No obstante, es necesario seguir trabajando en la validación para elementos estructurales reales de mayor tamaño para una aplicabilidad confiable en el análisis de daño tolerable.

En el desarrollo del ensayo de integral $J$ no se ha puesto en evidencia el crecimiento estable de fisura previo a la instabilidad. Al alcanzar el valor crítico la inestabilidad de la probeta es verificada, en forma completa, sin posibilidades de recuperación del proceso monótono de carga.

Se cuantifica la influencia en las propiedades mecánicas y de la cantidad de capas en la secuencia de apilamiento del laminado, siendo que, el compuesto elaborado con aluminio de mayor resistencia conserva esas características, a la vez que el aumento del número de capas también incrementa la resistencia y la tenacidad a la fractura.

\section{AGRADECIMIENTOS}

Los autores agradecen al personal técnico del Departamento de Materiales UAM-CNEA, Argentina. 


\section{BIBLIOGRAFÍA}

[1] VOGELESANG, B., "Development of new hybrid material (ARALL) for aircraft structure", Chemical Production Resourses Development, v.22, pp. 492-496, 1983.

[2] PALKOWSKI, H., LANGE, G., "Creation of tailored high strength hybrid sandwich structures", Advanced Materials Research, v. 22, pp. 27-36, Aug. 2007.

[3] LIN, C., KAO, P., "Effect of fiber bridging on the fatigue crack propagation on carbon fiber reinforced aluminum laminates", Materials Science \& Engineering, v. 190, pp. 65-72, Jan. 1995.

[4] MACHERET, Y., BUCCI, R., KULAK, M., "Metal plasticity and specimen size effects in evaluation of ARALL laminates notched panel residual strength", In: Proceedings 8th European Conference on Fracture, pp. 288-295, 1990.

[5] JIN, Z., BATRA, R., "Residual strength of centrally cracked metal/fiber composite laminates", Materials Science and Engineering, v. A216, pp. 117-124, 1996.

[6] ASTM- E561-05, "Standard test methods for K-R curve determination", Annual Book of ASTM Standards, American Society for Testing and Materials, 2005.

[7] CASTRODEZA, E., IPIÑA, J., BASTIAN, F., "Experimental techniques for fracture instability toughness determination of unidirectional fiber- metal laminates", Fatigue \& Fracture of Engineering Materials and Structures, v. 25, n.11, pp. 999-1008, 2002.

[8] ASTM- E1820-01, "Standard test methods for measurement of fracture toughness", Annual Book of ASTM Standards, American Society for Testing and Materials, 2001.

[9] RÍOS, J., CHOMIK, E., BALDERRAMA, J., et al., "Determination of fracture toughness J on fiber-metal laminate type CARALL with aluminum 6061”, Procedia Materials Science, v. 9, pp 530-537, 2015.

[10] LIN, C., KAO, P., YANG, F., "Fatigue behavior of carbon fiber reinforced aluminum laminates", Composites, v. 22, n. 2, pp. 135-141, 1991.

\section{ORCID}

Eduardo Asta

Francisco Adrián Cambiasso

Juan José Balderrama

Juan Carlos Rios https://orcid.org/0000-0002-2537-2340

https://orcid.org/ 0000-0002-7552-6037

https://orcid.org/ 0000-0001-7831-6783

https://orcid.org/0000-0002-8582-4513 